\title{
Visual Response Latencies in Temporal Lobe Structures as a Function of Stimulus Information Load
}

\author{
Kerry L. Coburn and J. Wesson Ashford \\ Department of Psychiatry \\ Southern Illinois University \\ School of Medicine, Springfield \\ Joaquin M. Fuster \\ Department of Psychiatry \\ University of California, Los Angeles
}

\begin{abstract}
In a monkey performing a visual delayed matching-to-sample task, units and visual evoked potentials (VEPs) were sampled from the inferior bank of the superior temporal sulcus (STS; Areas TEa and IPa), the hippocampus, and the presubiculum. VEP latencies indicated that flash information-signaling the imminent presentation of a color sample to be retainedreached the presubiculum and the hippocampus substantially earlier than the STS. In contrast, color sample VEP latencies did not differ between sites, arriving at all sites appreciably later than flash VEPs. Unit data indicated generally excitatory responses to both stimuli at all sites and net inhibition during the interstimulus interval separating flash from sample. As with VEPs, unit latencies to flash were shorter than to sample stimuli. The alerting flash data imply activation of the hippocampus occurring before activation of the STS cortex, whereas the coincident arrival of color sample information suggests temporal synchronization between these structures.
\end{abstract}

In attempting to understand the cortical mechanisms underlying visual perception and memory, considerable interest has focused on the inferotemporal cortex (ITC) and its evident role in analyzing and retaining information concerning behaviorally significant aspects of the visual environment. Earlier studies reported that single neurons of the ITC respond to behaviorally relevant dimensions of visual stimuli (Baylis \& Rolls, 1986), manifest elevated firing rates across a retention interval, and immediately cease responding after the discriminative behavioral response has been completed (Fuster \& Jervey, 1981, 1982).

Ashford and Fuster (1985) recorded unit activity and visual evoked potentials (VEPs) from the convexity of the inferotemporal cortex (Area TEm of Seltzer \& Pandya, 1978) during a visual delayed matching-to-sample (DMS) task. A large proportion of TEm neurons were inhibited by the warning flash preceding each trial but also showed an excitatory response to onset of the colored visual sample. Ashford and Fuster suggested that the flash response pattern might represent a suppression of TEm neurons not involved in processing alerting visual information, whereas the unit activation response reflected the processing of color information requiring discrimination and retention. The cortical area examined by Ashford and Fuster lay on the convexity of the middle temporal gyrus, immediately ventral to the superior

We thank Jerry Colliver and Steve Markwell for their assistance in analyzing the data presented in this article.

Correspondence concerning this article should be addressed to Kerry L. Coburn, Brain Mapping Laboratory, Department of Psychiatry, Southern Illinois University, School of Medicine, P.O. Box 19230, Springfield, Illinois 62794-9230. temporal sulcus (STS). Electrode penetrations were roughly perpendicular to the cortical surface, and unit activity was recorded to a depth of about $1.9 \mathrm{~mm}$. This article, using the same task, extends this study by sampling inferior bank neuronal activity and locally generated visual evoked potentials from Areas TEa and IPa (Seltzer \& Pandya, 1978) and the immediately subjacent hippocampal formation and presubiculum. To the extent that the hippocampal formation and presubiculum are involved in behaviorally relevant aspects of the DMS task, these neurons might be expected to exhibit response patterns related to those seen in the ITC.

\section{Method}

\section{Subject}

All recordings were made from 1 of the 2 male rhesus monkeys (Animal OH5; $8 \mathrm{~kg}$ ) described in Ashford and Fuster (1985). The monkey had ad lib access to food but had restricted access to water during the experimental period.

\section{Behavioral Paradigm}

The monkey was trained to perform a visual delayed matching to-sample task as described previously (Ashford \& Fuster, 1985). Briefly, each trial began with an alerting flash $(10 \mu \mathrm{s} ; 13 \mathrm{~lx}$ measured at the eyes) delivered through a white translucent rectangular $(22 \times$ $45 \mathrm{~cm}$ ) panel in the upper hemifield of the animal. The flash was followed $2 \mathrm{~s}$ later by red or green illumination (approximately 13.7 $\mathrm{cd} / \mathrm{m}^{2}$ ) of a stimulus-response button subtending $8^{\circ}$ of visual angle in a lower opaque panel. This sample stimulus lasted $1.5 \mathrm{~s}$; the animal had to acknowledge its presence by pressing the button within $1.0 \mathrm{~s}$ of its appearance. A 10-s delay period followed, at the end of which the two colors, red and green, appeared simultaneously in two but- 
tons placed side by side under the now unlit sample button. The monkey had to press the button showing the color of the previously presented sample. A correct match was reinforced by a squirt of liquid; an incorrect response immediately began the 30 -s intertrial interval. The sample color and its position in the lower buttons were changed at random from trial to trial.

\section{Surgical Procedure}

Under pentobarbital anesthesia a stainless steel electrode well was implanted over the inferotemporal cortex (area TEm) of the left temporal lobe. The electrode well was attached to the skull using screws and dental acrylic. Metallic sleeves also imbedded in the acrylic allowed fixation of the monkey's head during testing. Bicillin was administered systemically at regular intervals, and wound margins were treated with hydrogen peroxide and furacin ointment. The monkey was allowed to recover for several weeks prior to retraining and recording.

\section{Recording}

All recordings in this study were made using glass-coated elgiloy microelectrodes (Ashford, Coburn, \& Fuster, 1985). By using very small tip exposures, electrode impedances were set at $1 \mathrm{M} \Omega$ to simultaneously record single units and locally generated VEPs. Although much lower impedances are generally used for VEP recording, the combination of small tip exposure and high impedance in this study helped ensure that recorded VEPs represented locally generated activity. A stainless steel microelectrode positioner attached to the electrode well allowed penetration of the underlying dura and cortex at any point on a $2.5-\mathrm{mm}$-diameter circle. The electrode was advanced through the cortex and deeper structures by means of a microdrive from which depth measurements were taken for all recording sites. On-line analysis of neuronal activity gave an indication of responsivity to the stimuli of the task within a few trials. A total of 20 electrode penetrations to various depths along parallel tracks yielded 53 responsive units ( 25 in the STS; 21 in the hippocampus; 6 in the presubiculum). Recorded unit and VEP activity were bandpassed 30-3000 and $0.1-100 \mathrm{~Hz}$, respectively, through two Grass P511 differential amplifiers using the electrode well as the reference. Data were led through an A/D converter (VEPs) or Schmitt trigger (units) into a Radio Shack TRS-80 Model III microcomputer (1-kHz sampling rate) for analysis.

In previous recordings from this same animal, Ashford and Fuster (1985) sampled units on the convexity of the middle temporal gyrus (TEm). In this study the electrode carrier was positioned to allow electrodes to penetrate and record from the inferior bank of the STS traveling within the cortex roughly parallel to the cortical surface through Areas TEa and IPa.

Periods without unit activity were associated with electrode positions in the white matter. Deep electrode penetrations crossed the temporal horn of the lateral ventricle and penetrated the hippocampus and presubiculum. Also in contrast to the previous work, recording was scrupulously limited to isolated single units; multiple unit records were not taken. Isolation was continuously monitored by simultaneously displaying the output of a Schmitt trigger along with the unit activity. An adjustable threshold level for the Schmitt trigger with a minimum criterion of $2: 1$ signal/noise ratio ensured isolation. The principal reasons for recording local VEPs were to estimate the latencies of information arrival at each region (onset and first peak latencies) and to provide a sensitive indicator to validate electrode tip position with respect to tissue layers (waveform transitions).

\section{Histology}

At the end of this study the monkey was deeply anesthetized with pentobarbital, and electrolytic marking lesions were made at various depths. The monkey was then sacrificed and the brain removed and fixed in formalin. Coronal sections $(80 \mu \mathrm{m})$ were mounted, Nissl stained, and examined microscopically to verify electrode tracks, marking lesions, and recording sites.

\section{Data Analysis}

The behavioral accuracy of this highly trained monkey was over $95 \%$ correct and very stable across days. The rare incorrect responses were often associated with an obvious distraction or other procedural error, and for these reasons no attempt was made to separately analyze incorrect trials.

Visually responsive units were sampled from the cortex of TEa and IPa at various depths, from the hippocampal formation, and from the presubicular cortex. Locally generated VEPs and simultaneously recorded unit activity were averaged over blocks of 50 trials. Unit and VEP data (neuronal firing frequency and field voltage) were analyzed in 4-ms bins. Statistical analysis was conducted in two stages.

Stage 1 analysis selected only neurons that were responsive to the stimuli used in the DMS paradigm. In Stage 1, frequency histograms of unit activity were computed, and a unit was defined as responsive if its poststimulus firing frequency deviated from its mean $(500 \mathrm{~ms})$ preflash or presample baseline level in at least two successive bins by at least three standard deviations $(p=.001)$. Units not meeting this statistical criterion for responsiveness were dropped from the analysis along with the simultaneously recorded VEP data from that electrode tip position. No precise record was kept of the number of discarded units, but roughly half of the isolated units in all areas met these statistical criteria. (The common observation that about half of the units in a responsive cortical region are actuated by a behaviorally relevant stimulus is consistent with the view that information processing is based on spatio-temporal statistical patterns of activity within a functional neuronal ensemble [John, 1967, 1973] rather than the response of any specific unit.) Latencies for both units and their associated VEPs were measured after three-point binomial smoothing. Both unit and VEP onset latencies (time to the second consecutive bin differing by at least three standard deviations from the baseline average) and first peak latencies (time to the first frequency or voltage reversal point after the beginning of a significant response) were measured. Histograms and average VEP waveforms were computed at this stage.

In Stage 2 analysis, latency data from responsive units and VEP sites (i.e., data meeting the statistical criteria of Stage 1 analysis) were evaluated by two $7 \times 2 \times 2$ split-plot analyses of variance (ANOVAS), one on onset latency and the other on peak latency. The ANOVAS were used to determine the effects of brain level (shallow STS, middle STS, deep STS, shallow hippocampus, middle hippocampus, deep hippocampus, and presubiculum), stimulus (flash and sample), and sample color (red and green). Each ANOva had one between factors (brain level) and two within factors (stimulus and sample color). Error terms from the overall ANOvas were used to perform simple effects tests and follow-up tests using one-way ANOVAS with Duncan's multiple range tests and $t$ tests. Results were considered statistically significant if $p$ values were less than .05 .

\section{Results}

Reconstruction of the parallel electrode tracks and recording sites from serial sections showed that they lay within a relatively narrow cylinder of tissue (Figures 1 and 2). The 


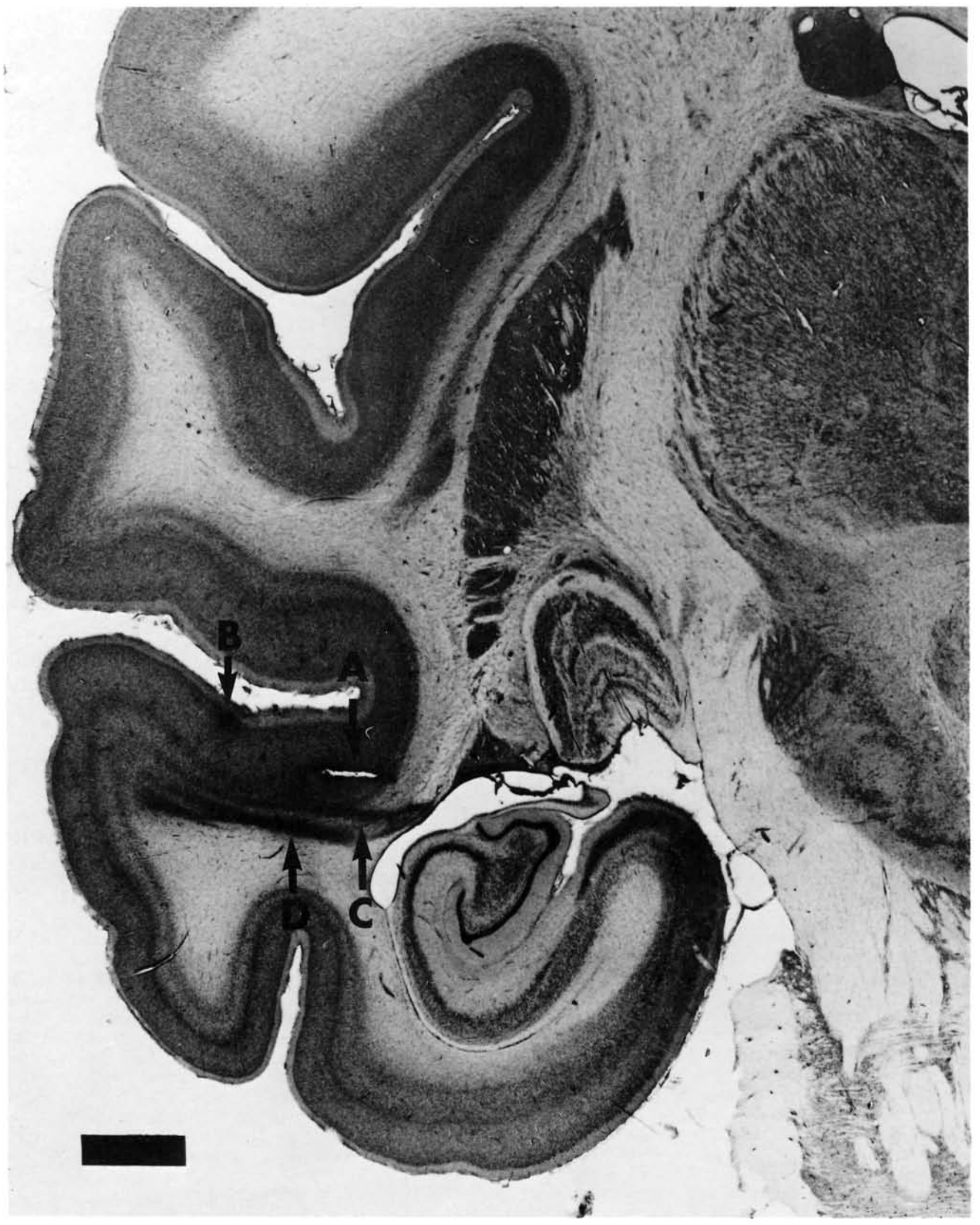

Figure 1. Coronal section through the left superior temporal sulcus showing cortical electrode track (A), marking lesion (B), hippocampal and presubicular electrode tracks $(\mathrm{C}$ and $\mathrm{D})$. $(\mathrm{Bar}=2.5 \mathrm{~mm}$.) 


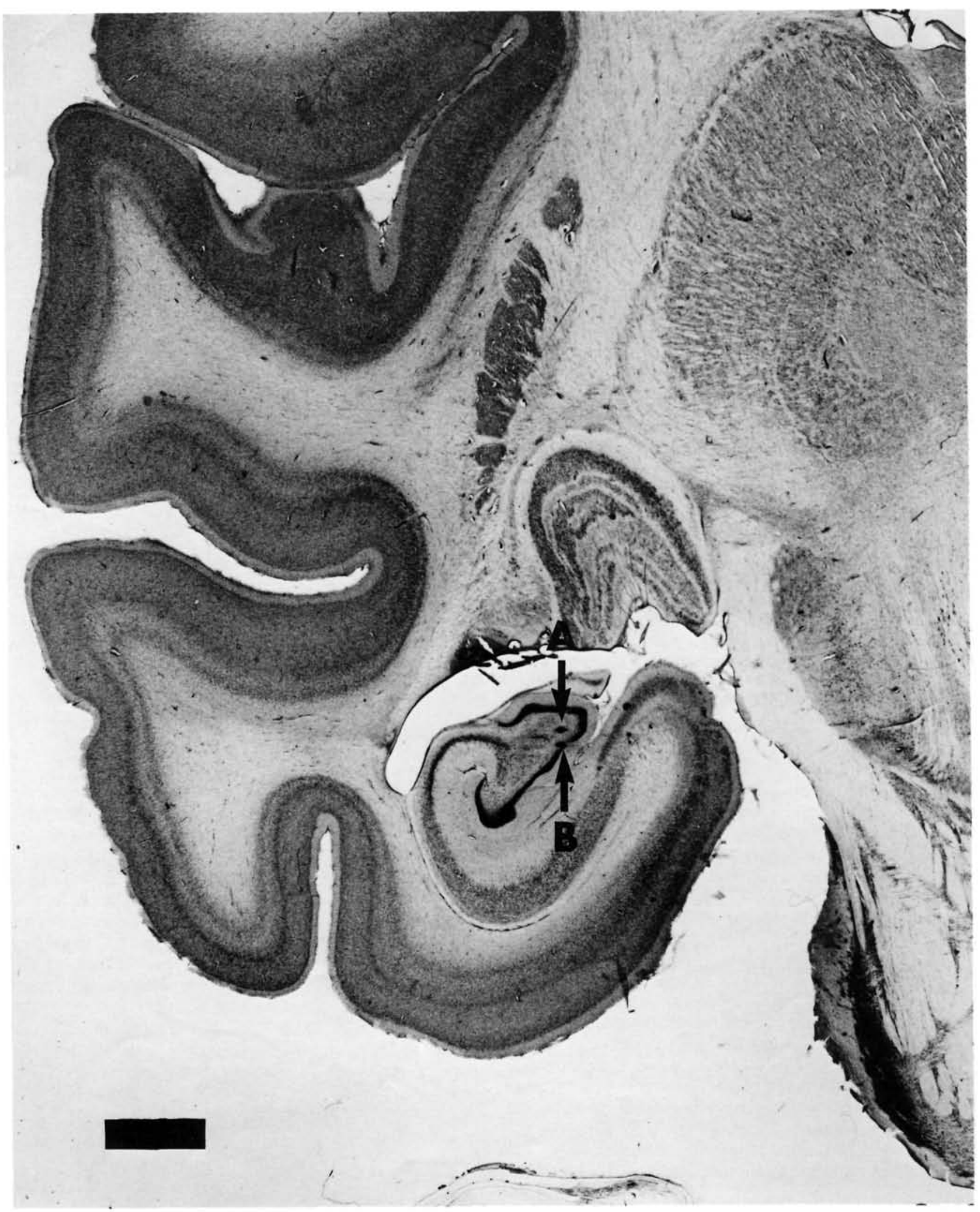

Figure 2. Coronal section through hippocampal formation showing electrode tracks $(\mathrm{A}$ and $\mathrm{B}) .(\mathrm{Bar}=2.5 \mathrm{~mm}$.) 


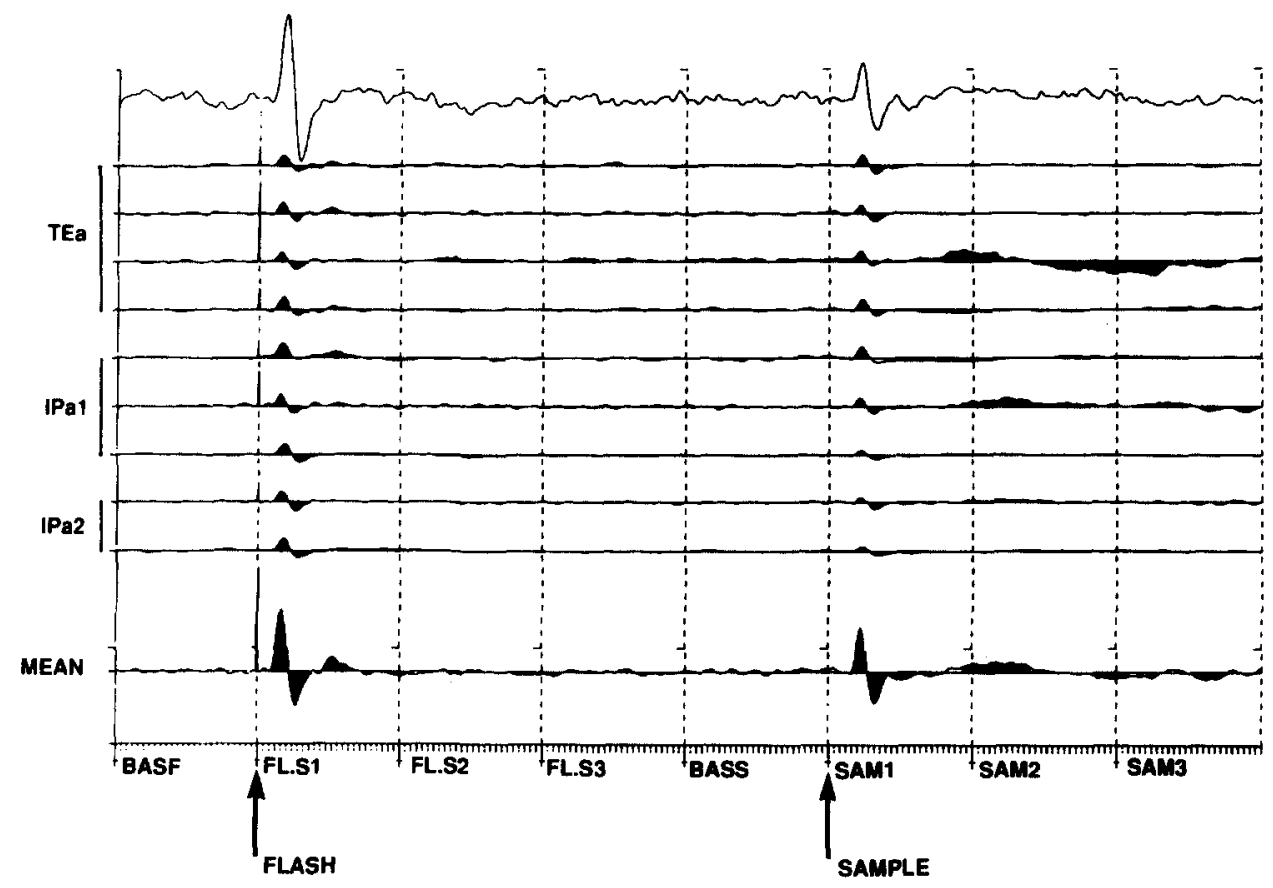

Figure 3. Averaged visual evoked potentials at various depths along the inferior bank of the superior temporal sulcus (STS). (Top trace is mean of raw data recorded from the middle depths of the STS. Middle traces are the output of Stage 1 data analysis, darkened to show statistical significance of amplitude change [ $>3$ standard deviations from preceding $500-\mathrm{ms}$ baseline, $p=.001]$ and scaled to a uniform height. The first evoked potential [EP] in each trace is the response to the trial-start flash, and the second EP is the response to the color sample presented $2 \mathrm{~s}$ later. The bottom trace is the rescaled mean of the nine preceding traces. Small tick marks on the horizontal axis show 20-ms increments; dotted lines are 500-ms increments. Depths of recording sites from the STS convexity are as follows: Area TEa, 1.0-5.5 mm; Area IPa [1], 5.6-7.0 mm; and Area IPa [2], 7.1-8.5 mm. Voltage deflections are positive up.)

electrode tracks ran parallel to the cortical surface down the inferior bank of the STS, across the temporal horn of the lateral ventricle, and then through the hippocampus and presubiculum. White matter positions were not associated with unit activity and were not used for VEP measurements. Unit and VEP data were recorded from 53 individual sites meeting Stage 1 responsiveness criteria.

\section{Inferotemporal Cortex}

Passing through Areas TEa and IPa along the lower cortical bank of the STS parallel to the cortical surface, 25 responsive single units meeting Stage 1 analysis criteria were isolated. Locally generated VEPs were recorded simultaneously with the unit action potentials. To assess whether response characteristics changed as a function of cortical depth within the STS, we subdivided the recordings into three groups on the basis of recording depth. Shallow cortical sites corresponded to Areas TEa, whereas middle and deep sites fell within Area IPa.

Response to alerting flash. Stage 1 analysis revealed that flash VEPs were of similar morphology throughout the depth of the sulcus, consisting of distinct P80, N140, and more variable P260 components. ${ }^{\top}$ The morphological stability of the VEP is consistent with the relatively constant geometric relationship between the equivalent dipole layers of the cortical pyramidal neurons, the electrode tip moving parallel to the cortical surface, and the orthogonally oriented electrode well reference. Examples recorded from representative sites within Areas TEa and IPa (filled to indicate intervals of statistical significance in Stage 1 analysis and scaled to a uniform amplitude) are shown in Figure 3. It can be seen in these data that the $\mathrm{P} 260$ tends to be more prominent in the more lateral (TEa) portions of the STS.

Examination of unit data (Stage 1 analysis) revealed that 23 of the 25 responsive units responded to the alerting flash, whereas the remaining 2 responded only to the sample. These responses were in most cases excitatory, although 3 units showed purely inhibitory responses to the flash, and 4 units

\footnotetext{
${ }^{1}$ Throughout this article positive $(\mathrm{P})$ and negative $(\mathrm{N})$ voltages recorded at the electrode tip with respect to the electrode well are distinguished with their corresponding peak latencies in milliseconds. Such designations do not necessarily imply homologies with human evoked potentials components.
} 


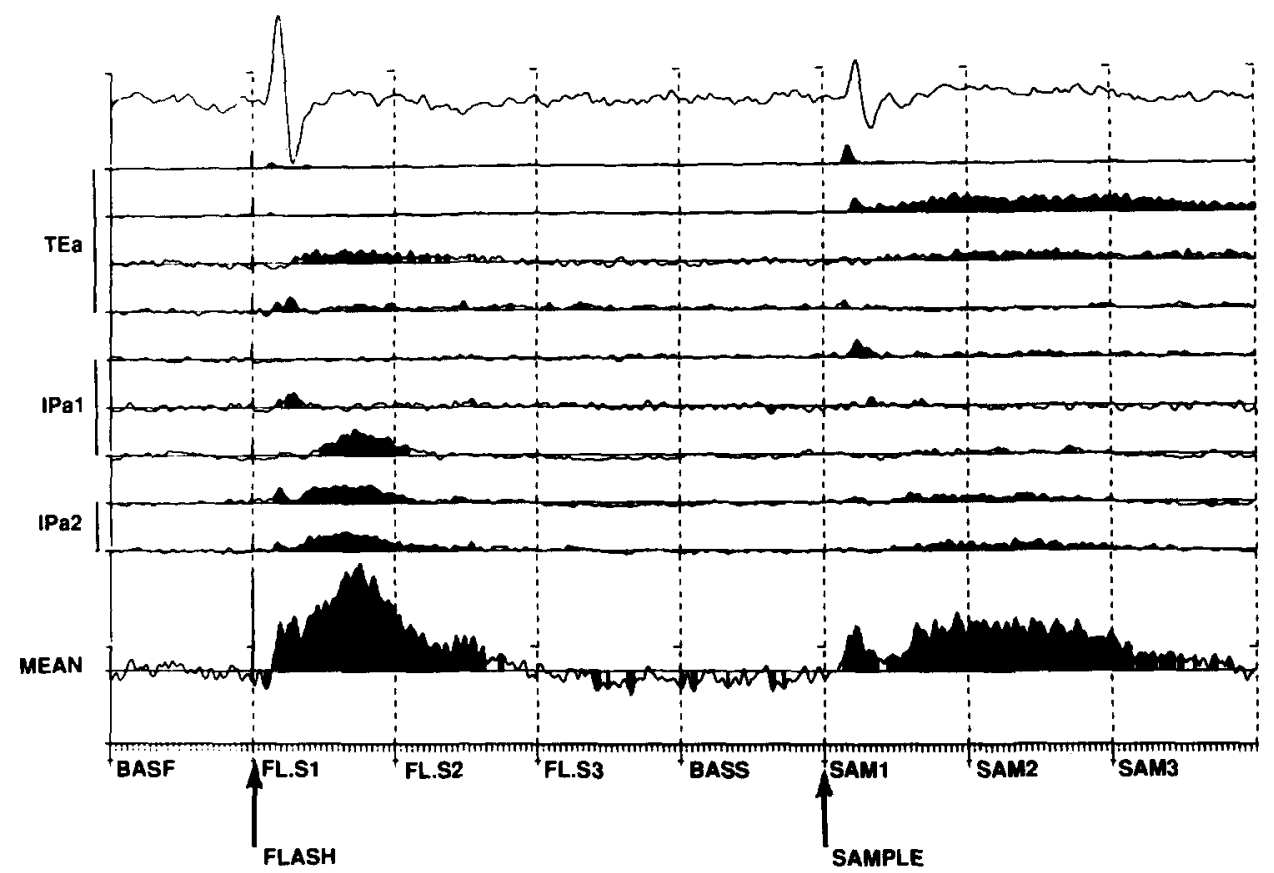

Figure 4. Average frequency histograms from isolated single neurons recorded simultaneously with the visual evoked potential (VEP) data shown in Figure 3. (Top trace is the mean of raw VEP data recorded from the middle depths of the superior temporal sulcus, shown for latency comparisons. Middle traces are the output of Stage 1 analysis, darkened to show statistically significant changes in firing frequency [ $>3$ standard deviations from preceding 500-ms baseline, $p=.001$ ] and scaled to a uniform height [thus disregarding absolute frequency differences between units]. The bottom trace is the rescaled mean of the nine middle traces. Note the tendency for unit activity to be inhibited during the interval separating the trial-start flash from the color sample presentation.)

showed mixed phases of excitation and inhibition. Examples recorded from representative sites within Areas TEa and IPa of the STS are shown in Figure 4 (same sites as in Figure 3). The onset and first peak of statistically significant unit activity change typically coincided with the rising wavefront of the local VEP. In the previous study (Ashford \& Fuster, 1985) a purely inhibitory unit response to the alerting flash was recorded from $36 \%$ of the neurons on the convexity of the middle temporal gyrus (TEm) of this monkey, whereas $41 \%$ showed a complete absence of significant response. In this study, only $12 \%$ and $8 \%$ of the sample-responsive units within the STS (Areas TEa and IPa combined) were found to fall into these two respective categories. There appeared to be a general trend toward proportionally increasing responsiveness to flash and decreasing responsiveness to color stimuli as recording electrodes penetrated into the depths of the STS progressing from Area TEa to Area IPa.

Activity during flash-sample interval. Largely because of the $0.5-\mathrm{Hz}$ lower bandpass roll-off, VEP data did not show a consistent pattern of statistically significant effects of the flash during the 2-s interstimulus interval (Figure 3). The single unit data (Figure 4) showed mixed effects during that interval, with a slight predominance of significant inhibition. Predominant inhibition of unit activity during that period was also observed in the convexity of the middle temporal gyrus of this monkey by Ashford and Fuster (1985).
Response to color sample. As shown in Figure 3, the sample VEP had a simple P120-N160 morphology with a negative component (N260) tending to appear at deep STS sites.

Single unit responses to the color sample were also recorded from 24 of the 25 responsive STS sites. As in the case of flash unit responses, most ( 22 of 25 ) were excitatory, and only 2 were inhibitory. Early excitation followed by much later inhibition was seen in only 1 unit. Although many units showed a larger response to one color than to the other, none was found to demonstrate color opponency. The representative units in Figure 4 show the variety of transient (first unit), sustained (second unit), and delayed (third unit) responses encountered. The types, latencies, and proportions of responses to the color sample of these isolated STS units were indistinguishable from those recorded on the convexity (TEm) of this monkey by Ashford and Fuster (1985).

\section{Hippocampus and Presubiculum}

Electrodes traversed the inferior horn of the lateral ventricle to sample units in $\mathrm{CA} 2, \mathrm{CA} 3, \mathrm{CA} 4$, dentate, and presubiculum (Figure 2).

Responses to alerting flash. The flash VEPs recorded in the hippocampus differed markedly from those recorded in the inferotemporal cortex. After entering CA2 the prominent P80 component of the STS flash VEP was markedly reduced 


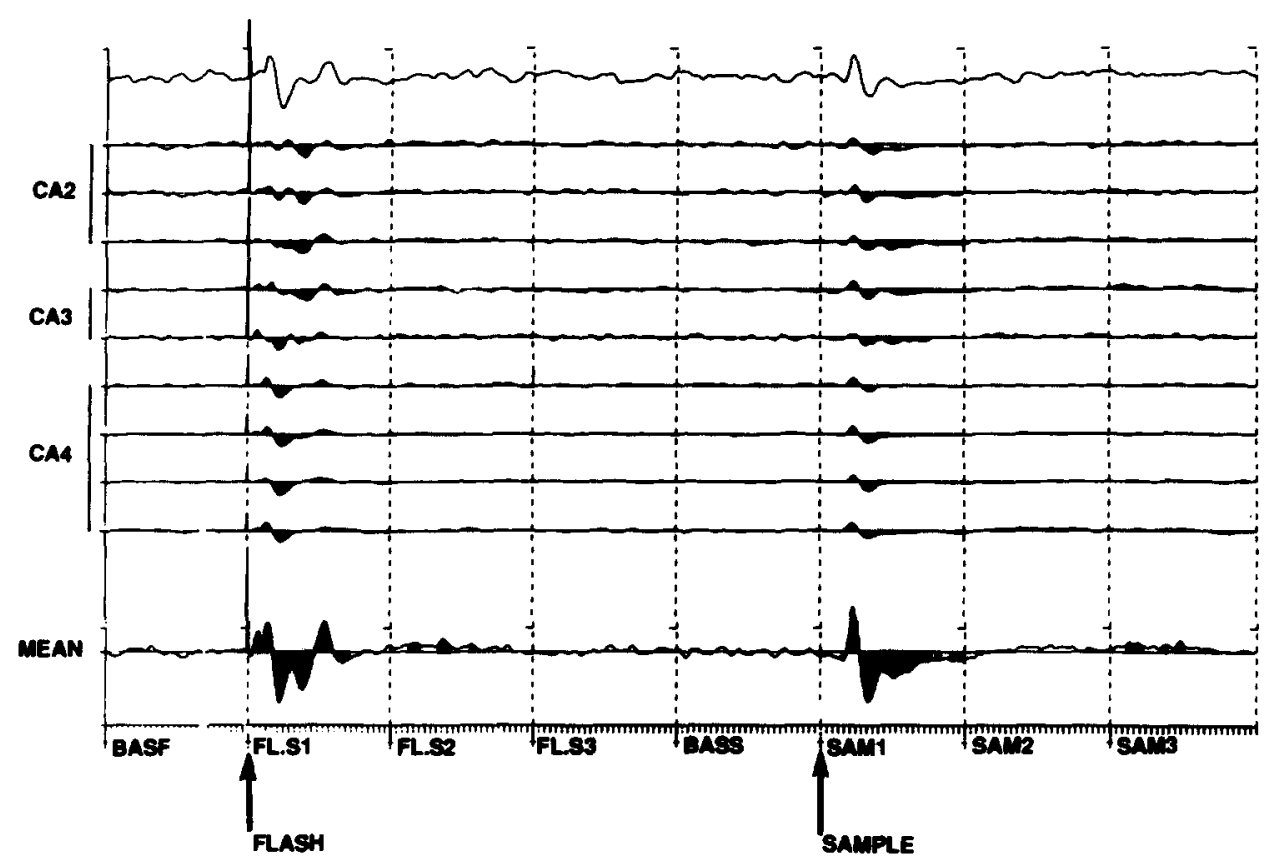

Figure 5. Visual evoked potentials (VEPs) from representative sites in the hippocampal formation. (Top trace is the mean of raw VEP data recorded from a site in CA4. Middle traces show the outcome of Stage 1 analysis for sites within CA2, CA3, and CA4 hippocampal areas [Truex \& Carpenter, 1969]. Bottom trace shows the rescaled mean of the eight middle traces. See captions of Figures 3 and 4 for details.)

and the N140 phase reversed (Figures 3 and 5). Flash VEPs underwent progressive changes through $\mathrm{CA} 3$ and CA4, examples of which are shown in Figure 5. Also, the hippocampal flash VEP contained more late components, including a P270 and a variable N340. The regionally specific morphology of the hippocampal flash VEP indicates a changing geometric relationship between the electrode tip and local equivalent dipoles, as the tip passes through, rather than along, pyramidal cell layers. Its complex morphology may also represent a decoupling of activity in this region from the overlying inferotemporal cortex. Immediately after entering the presubiculum (Figure 6), the initial positive component of the flash VEP was lost, and the ensuing negative component (N120) broadened.

A total of 21 responsive isolated single units were recorded in the hippocampus, and 6 units were recorded in the presubiculum (Figures 6 and 7). The flash response was predominantly excitatory with onset and first peak latencies increasing monotonically from the presubiculum to CA4 to $\mathrm{CA} 3$, and CA3 to CA2.

Activity during flash-sample interval. Flash VEPs from hippocampal sites tended to show a late positivity that was sporadically significant as late as $790 \mathrm{~ms}$ after the stimulus (Figure 5) before returning to baseline level. The unit activity showed sustained inhibition from about $800 \mathrm{~ms}$ and throughout the rest of the flash-sample interval (Figure 7). In the presubiculum, a statistically significant late VEP positivity was sustained throughout the flash-sample interval at most sites (Figure 6). Unit activity tended to return to baseline levels during this period, but at several sites in the presub- iculum and hippocampus, the firing rates of isolated units increased throughout the interval.

Response to color sample. Hippocampal VEPs to the color sample generally began with a small, short-latency (50$80 \mathrm{~ms}$ ), negative component that was of sporadic statistical significance (Figure 5). Large, stable P120 and N160 components were followed by a prolonged negativity lasting several hundred milliseconds. In the presubiculum, sample VEPs (Figure 6) showed a small and variable initial positive component followed by a larger broad negativity. Occasional slow sustained positive or negative VEP deflections extended well into the delay interval after the sample.

Unit activity in hippocampus and presubiculum showed generally excitatory responses to the sample (Figures 6 and 7). These responses tended to be prolonged and with a poorly defined configuration. In some instances they displayed a peaking of the elevated unit firing sustained throughout the flash-sample interval (as shown in the ninth traces of Figures 6 and 7).

\section{Latency Analysis}

The results of VEP latency analysis (Stage 2) are summarized in Figures 8 and 9 and in Table 1. For both onset and peak latencies, analyses showed no significant differences between red and green sample colors. Also, for both onset and peak latencies, a significant interaction of recording depth and stimulus was found $(p=.0024$ for onset; $p=.0019$ for peak), showing that brain locations did not differ in their VEP latencies to the sample stimulus $(p>.05)$ but that VEP 


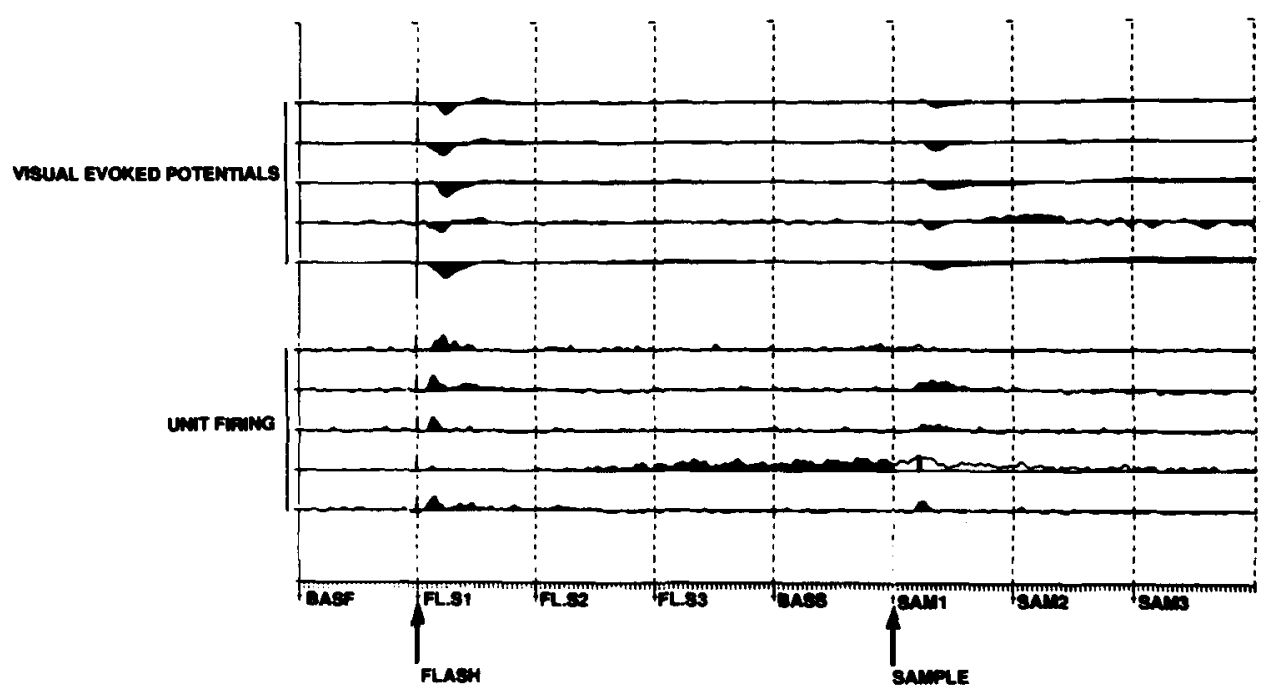

Figure 6. Composite of visual evoked potentials (top five traces) and unit action potentials (bottom five traces) recorded from sites in the presubiculum. (See captions of Figures 3 and 4 for details.)

latencies to the flash were shorter for deeper brain areas than for shallower areas $(p=.0545$ for onset; $p=.0109$ for peak).

Statistical follow-up tests (Table 1) showed that the flash VEP onset latencies of the three inferotemporal cortical levels (Areas TEa, IPa1, and IPa2) and the shallowest hippocampal level (Area CA2) differed significantly from the middle and deep hippocampus (Areas CA3 and CA4) and the presubiculum $(p<.05)$. For flash VEP peak latencies, these followup tests also showed that the two shallowest cortical levels differed significantly from the middle and deep hippocampal levels and the presubiculum $(p<.05)$. With the exception of the middle cortical level, the flash onset latency was also significantly shorter than the sample onset latency $(p<.05)$ at each depth. Also, for each level the flash peak latency was significantly shorter than sample peak latency $(p<.05)$.

The latencies of single unit responses to the task stimuli (Table 2) were more difficult to evaluate than those of VEPs, the reason being that responses of different units, even in the

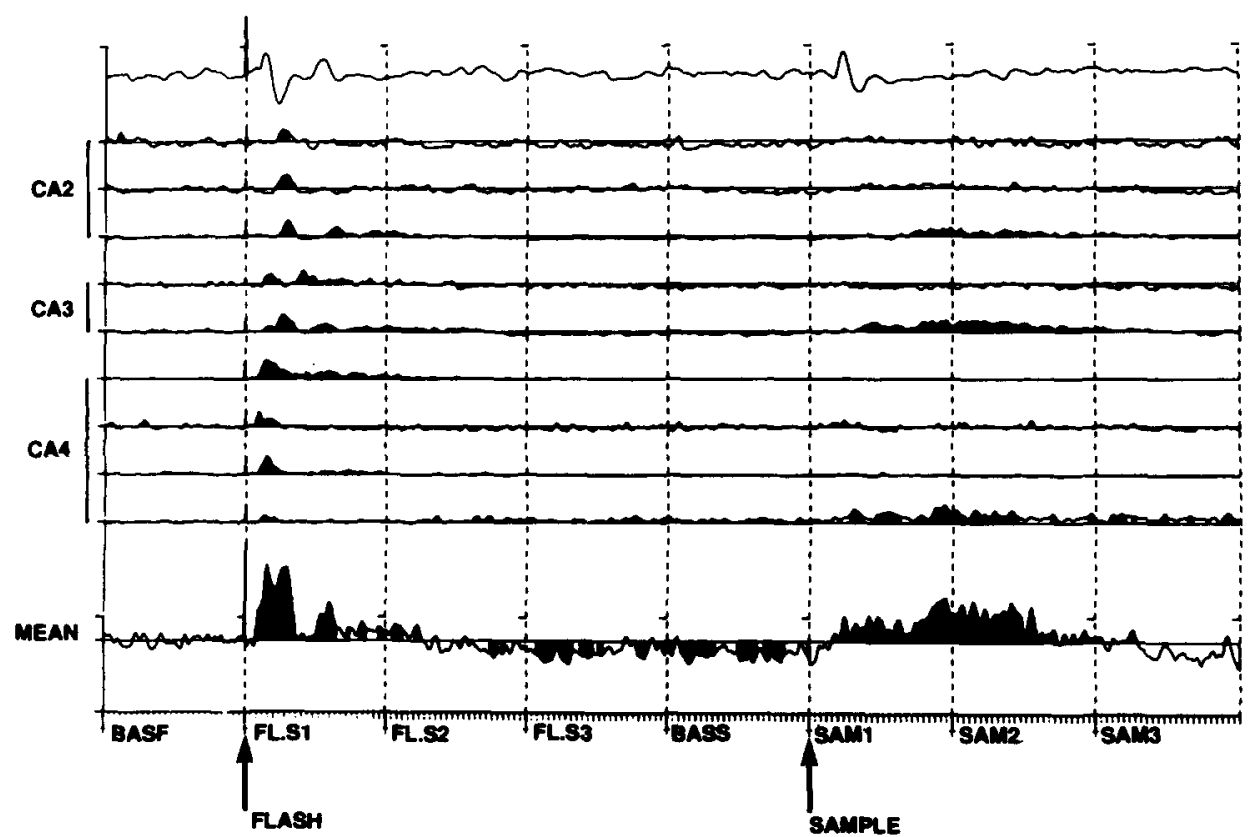

Figure 7. Average frequency histograms from isolated single neurons recorded simultaneously with the visual evoked potential (VEP) data shown in Figure 5. (Top trace is mean of raw VEP data recorded from a site in CA4 and is shown for latency comparisons. Middle traces are the output of Stage 1 analysis. The bottom trace is the rescaled mean of the eight middle traces. See captions of Figures 3 and 4 for details. As in the superior temporal sulcus cortex, note the tendency for unit activity to be inhibited during the interval separating the trial-start flash from the color sample presentation.) 


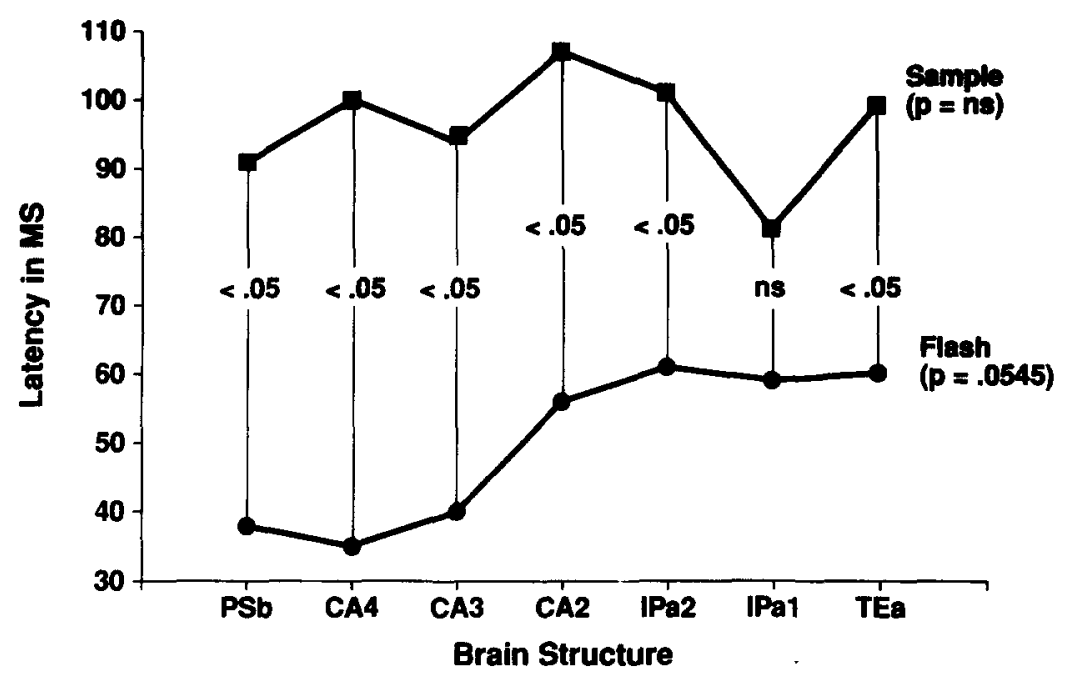

Figure 8. Onset latencies of visual evoked potentials recorded in each structure for trial-start and color sample stimuli. (See also Table 1.)

same region, varied considerably in the time domain. Overall, unit latencies for both flash onset $(p<.0008)$ and first peak $(p<.0011)$ were significantly shorter than those to the color sample. Latencies of unit responses to the flash (onset/ first peak) were also consistent with a direction of information flow from the presubiculum $(57 / 73 \mathrm{~ms})$ to CA4 $(68 / 80$ $\mathrm{ms}), \mathrm{CA} 4$ to CA3 (107/120 ms), and CA3 to CA2 (161/170 $\mathrm{ms})$.

\section{Discussion}

In a series of articles, Mishkin and his colleagues outlined a system of brain structures apparently underlying processing of visual detail in monkeys (Mishkin, 1982; Mishkin, Spiegler, Saunders, \& Malamut, 1982; Mishkin, Ungerleider, \& Macko, 1983). From perceptual and mnemonic performance deficits caused by large ablations, they inferred a cortical recognition system composed of a hierarchically arranged succession of neocortical areas extending along the middle temporal lobe, in association with the inferior longitudinal fasciculus, from the occipital pole to the temporal pole. These cortical areas sequentially process perceptual aspects of visual information related to object identification but not spatial location. After reaching Area TE in the inferotemporal cortex, there is anatomical and behavioral evidence that the information is further processed by interactions of that cortical area with the amygdala and hippocampus, interactions crucial for the storage of new visual information.

Using the technique of cortical cooling to produce reversible local depressions of Area TE, Fuster, Bauer, and Jervey (1981) found cooling-related deficits in visual recognition memory (DMS paradigm) but not in memory for spatial

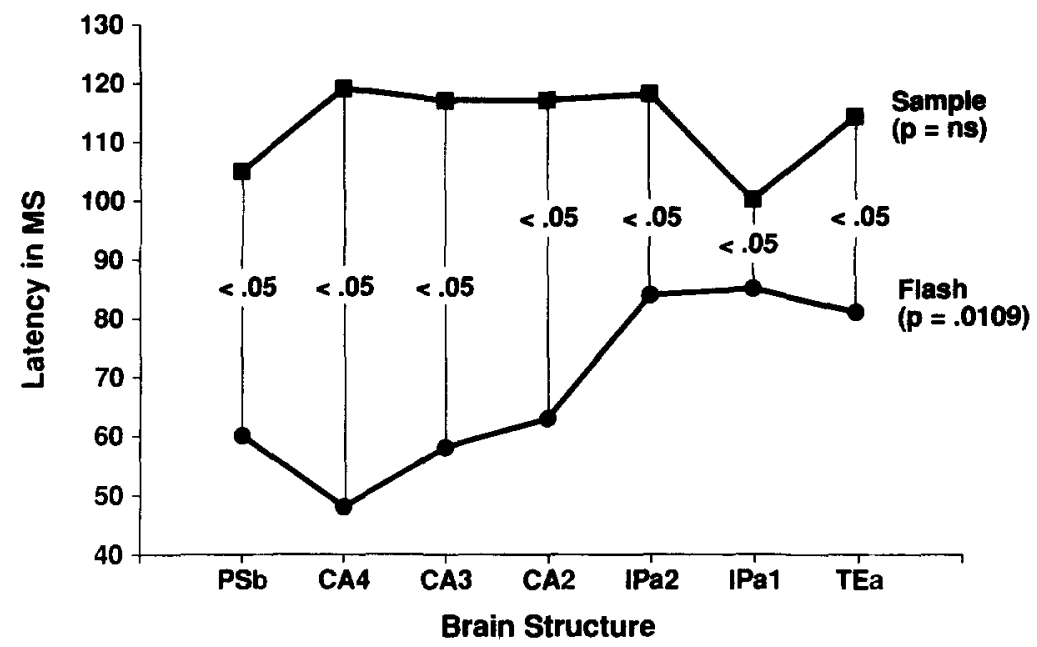

Figure 9. First peak latencies of visual evoked potentials recorded in each structure for trial-start and color sample stimuli. (See also Table 1.) 
Table 1

Visual Evoked Potential (VEP) Latencies

\begin{tabular}{|c|c|c|c|c|c|}
\hline \multirow[b]{2}{*}{$\begin{array}{l}\text { Recording } \\
\text { site }\end{array}$} & \multirow[b]{2}{*}{$n$} & \multicolumn{2}{|c|}{ Flash VEPa $^{a}$} & \multicolumn{2}{|c|}{ Sample VEPa } \\
\hline & & Onset $^{\mathrm{b}}$ & $\begin{array}{l}\text { First } \\
\text { peak }^{\mathbf{b}}\end{array}$ & Onset & $\begin{array}{l}\text { First } \\
\text { peak }\end{array}$ \\
\hline $\mathrm{TEa}$ & 9 & $60_{\mathrm{A}}$ & $81_{\mathrm{A}}$ & 99 & 114 \\
\hline $\mathrm{IPal}$ & 8 & $59_{\mathrm{A}}$ & $85_{\mathrm{A}}$ & 81 & 100 \\
\hline $\mathrm{IPa} 2$ & 8 & $61_{\mathrm{A}}$ & $84_{A B}$ & 101 & 118 \\
\hline $\mathrm{CA} 2$ & 7 & $56 \mathrm{~A}$ & $63_{\mathrm{BC}}$ & 107 & 117 \\
\hline CA3 & 7 & $40_{B}$ & $58_{\mathrm{c}}$ & 94 & 117 \\
\hline CA4 & 6 & $35_{\mathrm{B}}$ & $48_{\mathrm{C}}$ & 100 & 119 \\
\hline PSb & 6 & $38_{\mathrm{B}}$ & $60_{\mathrm{c}}$ & 91 & 105 \\
\hline
\end{tabular}

Note. $\mathrm{TEa}=$ shallow portion of inferior bank of superior temporal sulcus; $\mathrm{IPa}=$ deep portion of inferior bank of superior temporal sulcus; $1 \mathrm{~Pa} 1=$ superficial part of area IPa; $I \mathrm{~Pa} 2=$ deep part of area IPa; $\mathrm{CA} 2=$ hippocampal region CA2, shallow hippocampus; $\mathrm{CA} 3$ = hippocampal region CA3, middle hippocampus; $\mathrm{CA} 4=$ hippocampal region CA4, deep hippocampus; $\mathrm{PSb}=$ presubiculum. aMean latencies in milliseconds.

'Duncan's multiple range test: Means with same letter subscripts are not significantly different $(p<.05)$.

location (DR paradigm), thus supporting Mishkin's findings with irreversible lesions. The reversible cryogenic deficit in DMS performance has subsequently been confirmed by both intracortical (Horel \& Pytko, 1982) and epicortical (Horel, Voytko, \& Salsbury, 1984) cooling techniques.

In a study of a large number of monkey inferotemporal neurons, Baylis, Rolls, and Leonard (1987) characterized units in several architectonic areas in terms of their optimal stimulus requirements. In that study a very high proportion of neurons were found to be visually responsive in Areas TEm $(60 \%)$, TEa $(60 \%)$, and IPa (39\%), with maximal responsiveness in all areas to stationary stimuli. Although a wide variety of moving and stationary stimuli were used, unit response latencies were very similar to those found in this study (TEa and IPa) and in a previous study (TEm) with the same monkey (Ashford \& Fuster, 1985).

The activity of single inferotemporal neurons during behavioral performance of a DMS task suggests that they are involved in functional networks mediating the retention of behaviorally relevent, specific sample-stimulus information during the delay period (Baylis \& Rolls, 1986; Fuster \& Jervey, 1981, 1982). Such neurons are particularly prevalent in the lower bank of the STS and anterior aspects of TE. In order to study the temporal characteristics of unit activity at each end of the occipitotemporal chain of cortical processing areas during the visual DMS task, Ashford and Fuster (1985) recorded from neurons in the occipital cortex (Area OC) and on the convexity of the inferotemporal cortex (Area TEm). In the same paradigm as used in this study, the trial-start flash increased neuronal firing rates at occipital recording sites. At inferotemporal sites, on the other hand, the flash predominately had no effect or produced inhibition of unit activity. The color sample evoked increased unit firing in both occipital and inferotemporal regions. Both VEP latencies and unit latencies were found to be consistent with a reciprocally connected but simultaneously active series of visual processing stages of the type described by Mishkin and his coworkers (Mishkin, 1982; Mishkin et al., 1982; 1983). Those findings were interpreted as indicating that the occipitotemporal chain described by Mishkin et al. $(1982,1983)$ is activated selectively by stimuli requiring detailed analysis. The sequential arrangement presumably allows progressively higher orders of information analysis (Hayek, 1952).

This article extends the previous findings by recording at sites descending the lower bank of the superior temporal sulcus (TEa and IPa) and within the immediately subjacent hippocampal formation and the presubiculum. VEPs in response to the flash signaling the beginning of the DMS trial and the imminent presentation of the sample differ significantly in latency as a function of recording depth; flash information arrives at presubicular and hippocampal sites much earlier than at STS sites. This phenomenon may reflect a preferential activation of presubicular and hippocampal circuitry by a relevant alerting stimulus. In marked contrast to the trial-start flash, the onset and first peak latencies of the color sample VEPs do not differ significantly between structures. However, in all locations, the arrival of visual information about the color sample occurred at latencies greater than those for the flash. The simultaneous activation of those structures by the sample stimulus indicates that the occipital

Table 2

Unit Response Latencies

\begin{tabular}{|c|c|c|c|c|c|c|c|c|c|}
\hline \multirow{3}{*}{$\begin{array}{l}\text { Record- } \\
\text { ing site }\end{array}$} & \multirow[b]{3}{*}{$n$} & \multicolumn{4}{|c|}{ Flash unit } & \multicolumn{4}{|c|}{ Sample unit } \\
\hline & & \multicolumn{2}{|c|}{ Onset } & \multicolumn{2}{|c|}{ First peak } & \multicolumn{2}{|c|}{ Onset } & \multicolumn{2}{|c|}{ First peak } \\
\hline & & Mean $^{a}$ & Range $^{a}$ & Mean & Range & Mean & Range & Mean & Range \\
\hline TEa & 9 & 141 & $52-432$ & 153 & $60-432$ & 191 & $52-420$ & 197 & $56-420$ \\
\hline IPal & 8 & 120 & $12-232$ & 126 & $16-234$ & 195 & $20-432$ & 208 & $52-432$ \\
\hline $\mathrm{IPa} 2$ & 8 & 149 & $42-292$ & 165 & $90-292$ & 203 & $32-380$ & 207 & $32-380$ \\
\hline CA2 & 7 & 161 & $104-248$ & 170 & $108-238$ & 147 & 24-244 & 150 & $28-244$ \\
\hline $\mathrm{CA} 3$ & 7 & 107 & $62-200$ & 120 & $78-200$ & 181 & $80-316$ & 182 & $80-316$ \\
\hline CA4 & 6 & 68 & $16-204$ & 80 & $16-204$ & 152 & $96-312$ & 163 & $96-312$ \\
\hline PSb & 6 & 57 & $44-76$ & 73 & $68-80$ & 110 & $76-180$ & 122 & $72-188$ \\
\hline
\end{tabular}

Note. $\mathrm{TEa}=$ area $\mathrm{TEa}$, shallow superior temporal sulcus; $\mathrm{IPal}=$ superficial portion of area IPa, middle superior temporal sulcus; $\mathrm{IPa} 2=$ deep portion of area $\mathrm{IPa}$, deep superior temporal sulcus; $\mathrm{CA} 2=$ hippocampal region $\mathrm{CA} 2$, shallow hippocampus; $\mathrm{CA} 3=$ hippocampal region $\mathrm{CA} 3$, middle hippocampus; $\mathrm{CA} 4=$ hippocampal region $\mathrm{CA} 4$, deep hippocampus; $\mathrm{PSb}=$ presubiculum.

Mean latencies and ranges in milliseconds. 
cortex (Ashford \& Fuster, 1985), inferotemporal cortex, and hippocampus receive and analyze behaviorally relevant, specific color information, and this analysis is performed concurrently in all of these areas.

From a statistical standpoint the primary finding of this study is not simply that flash and sample VEPs have significantly different latencies (a main effect of stimulus type that could be due to differences in brightness, eye fixation, etc.) or simply that different brain areas receive visual information at significantly different latencies (a main effect of brain structure that could be due to differing connectional proximities) but rather a very significant first-order interaction between brain structure and stimulus type. This interaction implies that some factor other than stimulus type or brain structure exerts very reliable effects on VEP latency, and we suggest that this critical interaction factor has to do with the information conveyed by the stimuli.

The rapid channeling of trial-start flash information from the retina to the presubiculum (mean VEP latency of $38 \mathrm{~ms}$; mean unit latency of $57 \mathrm{~ms}$ ) agrees generally with the finding by MacLean, Yokota, and Kinnard (1968) of short-latency unit visual responses in the hippocampal gyrus (mean unit latency of $58.7 \mathrm{~ms}$ ). In MacLean et al. (1968), flashes were presented to a passive and lightly anesthetized monkey and contained no behaviorally relevant information; no visually responsive units were found in the hippocampus itself. In the present study the flashes were presented to an alert monkey and contained information about a subsequent event; hippocampal unit responses were found in CA4 and at somewhat longer latencies in $\mathrm{CA} 3$ and $\mathrm{CA} 2$, with hippocampal VEP latencies showing a similar pattern. After these initially excitatory responses, both hippocampal and STS units were inhibited throughout the remaining interstimulus period. Although it is difficult to make inferences across studies, our findings and those by MacLean et al. suggest that whether flash information accesses the hippocampal formation itself and its related circuitry depends on the flash's behavioral relevance to the organism.

The arrival of trial-start information indexed by flash VEPs at the presubiculum and hippocampus prior to its arrival at IPa or TEa implies that this information bypasses the multistep corticocortical processing chain described by Mishkin (1982) on its way to the hippocampus. Indeed, there would appear to be little reason for the simple flash information to undergo the same depth of visual processing required by the color sample information, which must be classified, retained, and recalled within each trial for correct performance. Trialstart information may arrive at the presubiculum and hippocampus through any of several projections from the brain stem to the cortex. A likely example is the pathway from the pulvinar, which receives strong visual input from the pretectum (Dineen \& Hendrickson, 1983) and from the retina directly (Mizuno, Itoh, Uchida, Uemura-Sumi, \& Matsushi$\mathrm{ma}, 1982$ ). The pulvinar establishes heavy projections to the entorhinal cortex during prenatal development in both monkeys and humans (Kostovic \& Rakic, 1984). The entorhinal cortex in turn communicates extensively with the presubiculum and hippocampus (Van Hoesen \& Pandya, 1975a, 1975b).
In marked contrast to the responses to the trial-start flash, the onset and first peak latencies of the VEP responses to the color sample did not differ significantly between structures. The similarity of response latencies in these visually responsive areas may represent the temporal synchronization of several cortical processing areas along the occipitotemporal pathway and hippocampal formation for analysis of the behaviorally relevant, specific color information in a hierarchical manner (Maunsell \& Van Essen, 1983). Coincident input to neurons in these regions may form the basis for a parallel distributed processing system (Edelman \& Mountcastle, 1978) and be essential for the encoding of information into memory (Lynch, Granger, Larson, \& Baudry, 1989; McNaughton, Douglas, \& Goddard, 1978).

\section{References}

Ashford, J. W., Coburn, K. L., \& Fuster, J. M. (1985). The elgiloy microelectrode: Fabrication techniques and characteristics. Journal of Neuroscience Methods, 14, 247-252.

Ashford, J. W., \& Fuster, J. M. (1985). Occipital and inferotemporal responses to visual signals in the monkey. Experimental Neurology, 9, 444-466.

Baylis, G. C., \& Rolls, E. T. (1986). Responses of neurons in the inferior temporal cortex in short term and serial recognition memory tasks. Experimental Brain Research, 7, 330-342.

Baylis, G. C., Rolls, E. T., \& Leonard, C. M. (1987). Functional subdivisions of the temporal lobe neocortex. Journal of Neuroscience, 7, 330-342.

Dineen, J. T., \& Hendrickson, A. (1983). Overlap of retinal and prestriate cortical pathways in the primate pretectum. Brain $R e$ search, 278, 250-254.

Edelman, G. M., \& Mountcastle, V. B. (1978). The mindful brain. Cambridge, MA: MIT Press.

Fuster, J. M., Bauer, R. H., \& Jervey, J. P. (1981). Effects of cooling inferotemporal cortex on performance of visual memory tasks. Experimental Neurology, 71, 398-409.

Fuster, J. M., \& Jervey, J. P. (1981). Inferotemporal neurons distinguish and retain behaviorally relevant features of visual stimuli. Science, 212, 952-955.

Fuster, J. M., \& Jervey, J. P. (1982). Neuronal firing in the inferotemporal cortex of the monkey in a visual memory task. Journal of Neuroscience, 2, 361-375.

Hayek, F. A. (1952). The sensory order. Chicago: University of Chicago Press.

Horel, J. A., \& Pytko, D. E. (1982). Behavioral effect of local cooling in temporal lobe of monkeys. Journal of Neurophysiology, 47, 11 22.

Horel, J. A., Voytko, M. L., \& Salsbury, K. G. (1984). Visual learning suppressed by cooling the temporal pole. Behavioral Neuroscience, 98, 310-324.

John, E. R. (1967). Mechanisms of memory. New York: Academic Press.

John, E. R. (1973). Switchboard versus statistical theories of learning and memory. Science, 181, 764-767.

Kostovic, I., \& Rakic, P. S. (1984). Development of prestriate visual projections in the monkey and human fetal cerebrum revealed by transient cholinesterase staining. Journal of Neuroscience, 4, 2542.

Lynch, G., Granger, R., Larson, J., \& Baudry, M. (1989). Cortical encoding of memory: Hypotheses derived from analysis and simulation of physiological learning rules in anatomical structures. In L. Nadel, L. A. Cooper, P. Culicover, \& R. M. Harnish (Eds.), 
Neural connections, mental computation (pp. 181-224). Cambridge, MA: MIT Press.

MacLean, P. D., Yokota, T., \& Kinnard, M. A. (1968). Photically sustained on-responses of units in posterior hippocampal gyrus of awake monkey. Journal of Neurophysiology, 31, 870-883.

Maunsell, J. H. R., \& Van Essen, D. C. (1983). The connections of the middle visual area (MT) and their relationship to a cortical hierarchy in the macaque monkey. Journal of Neuroscience, 3 , 2563-2586.

MeNaughton, B. L., Douglas, R. M., \& Goddard, G. V. (1978). Synaptic enhancement in fascia dentata: Cooperativity among coactive afferents. Brain Research, 157, 277-293.

Mishkin, M. (1982). A memory system in the monkey. Philosophical Transactions of the Royal Society of London, Series B, 298, 8595.

Mishkin, M., Spiegler, B. J., Saunders, R. C., \& Malamut, B. L. (1982). An animal model of global amnesia. In S. Corkin, K. L. Davis, J. H. Growden, E. Usdin, and R. J. Wurtman (Eds.), Alzheimer's disease: A report of progress (pp. 235-247). New York: Raven Press.

Mishkin, M., Ungerleider, L. G., \& Macko, K. A. (1983). Object vision and spatial vision: Two cortical pathways. Trends in Neuroscience, $6,414-417$.
Mizuno, N., Itoh, K., Uchida, K., Uemura-Sumi, M., \& Matsushima, R. (1982). A retino-pulvinar projection in the macaque monkey as visualized by the use of anterograde transport of horseradish peroxidase. Neuroscience Letters, 30, 199-203.

Seltzer, B., \& Pandya, D. N. (1978). Afferent cortical connections and architectonics of the superior temporal sulcus and surrounding cortex in the rhesus monkey. Brain Research, 149, 1-24.

Truex, R. C., \& Carpenter, M. B. (1969). Human neuroanatomy (pp. 532-533). Baltimore, MD: Williams \& Wilkins.

Van Hoesen, G. W., \& Pandya, D. N. (1975a). Some connections of the entorhinal (area 28) and perirhinal (area 35) cortices of the rhesus monkey: I. Temporal lobe afferents. Brain Research, 95, $1-24$.

Van Hoesen, G. W., \& Pandya, D. N. (1975b). Some connections of the entorhinal (area 28) and perirhinal (area 35) cortices of the rhesus monkey: III. Efferent connections. Brain Research, 95, 3959.

Received December 28, 1988

Revision received July 12, 1989

Accepted July 18, 1989

\section{Low Publication Prices for APA Members and Affiliates}

Keeping You Up-to-Date: All APA members (Fellows; Members; and Associates, and Student Affiliates) receive--as part of their annual dues--subscriptions to the American Psychologist and the APA Monitor.

High School Teacher and Foreign Affiliates receive subscriptions to the APA Monitor and they can subscribe to the American Psychologist at a significantly reduced rate.

In addition, all members and affiliates are eligible for savings of up to $50 \%$ on other APA journals, as well as significant discounts on subscriptions from cooperating societies and publishers (e.g., the British Psychological Society, the American Sociological Association, and Human Sciences Press).

Essential Resources: APA members and affiliates receive special rates for purchases of APA books, including the Publication Manual of the APA, the Master Lectures, and APA's Guide to Research Support.

Other Benefits of Membership: Membership in APA also provides eligibility for low-cost insurance plans covering life; medical and income protection; hospital indemnity; accident and travel; Keogh retirement; office overhead; and student/school, professional, and liability.

For more information, write to American Psychological Association, Membership Services, 1200 Seventeenth Street NW, Washington, DC 20036, USA. 